\title{
Incidence and predictors of lost to follow-up among women under Option B+ PMTCT program in western Ethiopia: a retrospective follow-up study
}

Tadesse Tolossa ${ }^{1 *}$, Getachew Mullu Kassa ${ }^{2}$, Habtamu Chanie ${ }^{2}$, Amanuel Abajobir ${ }^{3}$ and Diriba Mulisa ${ }^{4}$

\begin{abstract}
Objective: Although Ethiopia has been implementing Option B+ program, LTFU of women from the Option B+ program is one of the challenges that minimizes its implementation. Thus, this study assessed the incidence and predictors of LTFU among women under Option B+ PMTCT program in western Ethiopia. An institution-based retrospective follow-up study was conducted. A cox proportional hazards regression model was fitted to identify predictors of LTFU. A Hazard ratios with 95\% confidence Cl was computed and all predictors that were associated with the outcome variable at $\mathrm{p}$-value $\leq 0.05$ in the multivariable cox proportional hazards were declared as a significance predictor of the outcome.

Results: A total of 330 women were followed for a mean follow up time of $16.9( \pm 7.6)$ months. An overall incidence rate of LTFU was 9/1000 person-months. Women's educational status, residence, HIV-disclosure status, the status of women at enrollment, previous history of HIV and ART adherence were significant predictors of LTFU. The incidence of LTFU from Option B+ PMTCT is lower as compared to evidence from sub-Saharan African and strengthening linkage and referral system between clinics as well as establishing appropriates tracing mechanisms would retain pregnant women in the program.
\end{abstract}

Keywords: Lost, Option B+, Mother-to-child transmission, Ethiopia

\section{Introduction}

The number of new pediatric HIV infections was reduced from 270,000 in 2009 to 160,000 in 2017 worldwide, more than $90 \%$ of these children were infected through mother-to-child transmission (MTCT). That is, without intervention, the risk of MTCT is $15-30 \%$ during pregnancy and delivery and 5-20\% during breastfeeding contributing for an overall transmission rate of $20-45 \%$ [1]. In western and Central Africa, the rate of MTCT of HIV

\footnotetext{
*Correspondence: yadanotolasa@gmail.com

${ }^{1}$ Department of Public Health, Institute of Health Science, Wollega

University, P.O.BOX: 395, Nekemte, Ethiopia

Full list of author information is available at the end of the article
}

was $20.2 \%$, and around 180,000 children acquired HIV during delivery and breast-feeding in 2017 [2].

The World Health Organization (WHO) guideline on ART recommends three options to prevent MTCT transmission of HIV infection-Options $\mathrm{A}, \mathrm{B}$, and $\mathrm{B}+$. The latest approach, Option $\mathrm{B}+$ PMTCT program, emphasizes on the provision of universal, lifelong ART for all HIV-infected women regardless of CD4 count and WHO clinical staging [3]. Moreover, WHO developed guidelines recommending a 'treat all' approach, meaning all people diagnosed with HIV should be offered immediate treatment. This has increased the number of women of reproductive age who are receiving ART, regardless of whether they are pregnant or not [4]. 
Ethiopia has been implementing Option $\mathrm{B}+$ program since 2013 as part of its national policy for preventing new HIV infections among children and to improve maternal survival [5]. Under Option $\mathrm{B}+$, all HIV-infected pregnant women will receive universal ART and will continue the treatment for the rest of their live [6] and have an advantage of simplification of ART, protection against MTCT in future pregnancies, a continuing prevention benefit against sexual transmission to serodiscordant partners, avoiding "stop start-stop" approach of antiretroviral drugs and minimize the opportunity of LTFU [7]. Before the implementation of option B+ PMTCT program, Ethiopia is one of the top 20 countries, where one children infected with HIV out of three child born to women living with HIV [5]. The proportion of pregnant women living with HIV who received antiretroviral medicines for the prevention of mother-to-child transmission has increased from a baseline of 37\% in 2009 to $77 \%$ in 2014 and increased to $80 \%$ in 2017 and estimated 1.4 million infectious among children age $0-14$ years was decreased from 2010 to 2017 [8, 9]. This indicates a major success for the Option B+ strategy and how it enables pregnant women to get antiretroviral therapy.

In the meantime, retention of pregnant and lactating women on universal lifelong ART is one of the crucial success indicators of the Option $\mathrm{B}+$ program. This is particularly important to achieve optimal maternal health and successful PMTCT program throughout all stages of PMTCT services. However, loss to follow-up (LTFU) from the PMTCT program creates a great challenge for the successful implementation of option $\mathrm{B}+$ program particularly in sub-Saharan Africa (SSA) where the prevalence of HIV is high $[2,10]$.

Overall, different studies show that younger age, lack of education, stigma and discrimination, failure to disclose HIV-status, long distances to reach health facilities, long waiting times, poor adherence to treatment, high CD4 count and WHO clinical stage I, II were associated with LTFU [11-13].

Unfortunately, LTFU from Option B+ decreases women's access to HIV care and treatment, which leads to the advanced stage of HIV, increases maternal HIV/AIDSrelated morbidity and mortality, facilitates the vertical transmission of HIV to newborn and facilitates the development of drug resistance [10, 14]. Even though Option $\mathrm{B}+$ program has been implemented for the last 6 years, there are no studies and/or documented reports on the incidence of LTFU and its predictors among pregnant and lactating women on lifelong ART.

Therefore, this study aimed to assess the incidence and predictors of LTFU among women under the Option B+ program at Nekemte Specialized Hospital in western Ethiopia.

\section{Main text}

Methods

This study was conducted at Nekemte Specialized Hospital among women enrolled in Option B+ PMTCT program from 12 June 2013 through to December 1, 2018. The provision of ART services was initiated in 2005 in the hospital and the Option B+ PMTCT program started in 12 June 2013. A retrospective follow-up study was employed. From June 12, 2013 to December 1, 2018, 412 women were started ART under Option B+ PMTCT program at Nekemte specialized hospital. Of the total women who started ART under Option B+ program, 19 patients' cards were not available. Three hundred ninety-three patient cards were reviewed and 63 patient cards were excluded due to incompleteness of the data (date of HIV diagnosis, date of ART initiation, recent date and outcome not recorded). Finally, 330 patient cards with complete data were included in the final analysis.

\section{Study variables}

\section{Dependent variable}

Incidence of LTFU from Option B+ PMTCT.

\section{Operational definition}

Event: LTFU which is 3 months after the last documented visit under Option B+ PMTCT and not recorded as 'dead' or 'transferred-out' on patient PMTCT logbook or medical cards $[11,15,16]$.

Censored: a patient did not develop an event or LTFU (death, transferred-out, treatment completed, and receiving treatment when the study was ended).

Survival time: the time in months from the beginning of treatment under the Option B+ PMTCT program to LTFU from the program.

\section{Data collection, data management, and analyses}

The data were collected by using a checklist, developed from FMoH PMTCT logbook, ART intake forms and medical cards of eligible participants. The outcome variable, LTFU, was confirmed by reviewing the medical or PMTCT registration logbook at the hospital, which was recorded by health professionals working in the PMTCT clinic. Two trained nurses working at the PMTCT clinic were recruited for data collection, and one trained Public Health practitioner supervised the overall data collection.

Epidata version 3.2 was used for data entry, and then the data were exported to STATA version 14 for 
cleaning and further analyses. Descriptive non-parametric survival analyses such as the life-table and Kaplan-Meier survival curves were used for estimating survival probability.

To identify predictors of LTFU, bivariable and multivariable Cox regression analysis was conducted. To select variables for multivariable cox analysis, bivariable cox regression at $\mathrm{p}$-value $\leq 0.25$ were used. Then full multivariable cox analysis was conducted by using a backward stepwise selection process including all the potential risk factors that had a p-value of $\leq 0.25$ in bivariable Cox proportional hazard analysis. Then the final best model for multivariable proportional hazard analysis was selected by using Log likelihood ratio (LLH). Hazard ratios (HR) with $95 \%$ confidence intervals (CIs) were computed and statistical significance was declared when it is significant at a $5 \%$ level (p-value $<0.05$ ).

\section{Results}

Sociodemographic and maternal characteristics of study participants

During the study period, a total of 393 women were initiated ART under option B+ PMTCT program at Nekemte specialized hospital. Of them, 330 (84\%) women's chart was included in the analysis and $63(16 \%)$ charts were excluded due to incompleteness of the data. The mean ( \pm standard deviation (SD)) age of women was $26.6 \pm 4.5$ years. One hundred fifty $(45.4 \%)$ women belonged to 25-29 years' age group. The study revealed that 199 (60.0\%) study participants were urban residents, 217 (65.8\%) were married. (Table 1).

\section{Baseline clinical, laboratory and follow-up characteristics}

Of the total observations, 271 (82.1\%) of women were at WHO clinical stage I/II and $263(79.70 \%)$ had a CD4 count greater than 351 cells $/ \mathrm{mm}^{3}$ at baseline. The mean hemoglobin level at enrollment was $12.0 \mathrm{mg} / \mathrm{dl}$. The

Table 1 Baseline sociodemographic and maternal characteristics of women under Option B+ PMTCT program at Nekemte Specialized Hospital, western Ethiopia, 2013-2019

\begin{tabular}{|c|c|c|c|c|}
\hline \multirow[t]{3}{*}{ Variables } & \multirow[t]{3}{*}{ Category } & \multicolumn{2}{|c|}{ Survival status } & \multirow{3}{*}{$\begin{array}{l}\text { Total } \\
\text { No (\%) }\end{array}$} \\
\hline & & LTFU & Censored & \\
\hline & & No (\%) & No (\%) & \\
\hline \multirow[t]{3}{*}{ Age } & $\leq 24$ & $26(51.0)$ & $67(24.0)$ & $93(28.2)$ \\
\hline & $25-29$ & $17(33.3)$ & $133(47.7)$ & $150(45.4)$ \\
\hline & $\geq 30$ & $8(15.7)$ & $79(28.3)$ & $87(26.4)$ \\
\hline \multirow[t]{2}{*}{ Residence } & Rural & $34(66.7)$ & $97(34.8)$ & $131(39.7)$ \\
\hline & Urban & $17(33.3)$ & $182(65.2)$ & $199(60.3)$ \\
\hline \multirow[t]{3}{*}{ Marital status } & Never & $13(25.5)$ & $41(14.7)$ & $54(16.3)$ \\
\hline & Married & $30(58.8)$ & $187(67.0)$ & $217(65.8)$ \\
\hline & Widowed/divorced & $8(15.7)$ & $51(18.3)$ & $59(17.9)$ \\
\hline \multirow[t]{3}{*}{ Educational status } & No education & $22(43.1)$ & $63(22.6)$ & $85(25.8)$ \\
\hline & Primary & $19(37.3)$ & $94(33.7)$ & $113(34.2)$ \\
\hline & Secondary and above & $10(19.6)$ & $122(43.7)$ & 132(40.0) \\
\hline \multirow[t]{5}{*}{ Employment } & Housewife & $24(47.1)$ & $128(45.9)$ & 152(46.1) \\
\hline & Gov't employee & $10(19.6)$ & $74(26.5)$ & $84(25.5)$ \\
\hline & Non gov't employee & $4(7.8)$ & $33(11.8)$ & $37(11.2)$ \\
\hline & Daily laborer & $7(13.7)$ & $30(10.8)$ & $37(11.2)$ \\
\hline & Others & $6(11.8)$ & $14(5.0)$ & $20(6.0)$ \\
\hline \multirow[t]{3}{*}{ Partner HIV status } & Positive & $8(15.6)$ & $98(35.1)$ & $106(32.1)$ \\
\hline & Negative & $24(47.1)$ & $113(40.5)$ & $137(51.5)$ \\
\hline & Unknown & $19(37.3)$ & $68(24.4)$ & $87(26.4)$ \\
\hline \multirow[t]{2}{*}{ Disclosure status } & No & $29(56.9)$ & $56(20.1)$ & $85(25.8)$ \\
\hline & Yes & $22(43.1)$ & $223(79.9)$ & $245(74.2)$ \\
\hline \multirow[t]{2}{*}{ Status of women on enrollment } & Pregnant & $39(76.5)$ & $249(89.2)$ & $288(87.3)$ \\
\hline & Lactating & $12(23.5)$ & $30(10.8)$ & $42(12.7)$ \\
\hline \multirow[t]{2}{*}{ Number of pregnancy } & Single & $28(54.9)$ & $86(30.8)$ & $114(34.6)$ \\
\hline & Multiple & $23(45.9)$ & $193(69.2)$ & $216(65.4)$ \\
\hline
\end{tabular}


predominant regimens initially prescribed were a combination therapy-TDF/3TC/EFV for 240 (72.7\%) participants, followed by AZT/3TC/NVP for 51 (15.45\%). During the follow-up period, 165 (50\%) women took CPT at baseline and 306 (92.7\%) were negative for TB screening and $160(52.3 \%)$ took IPT.

\section{Survival status of women}

A total of 330 patients were followed for a mean time of 16.9 ( $\mathrm{SD} \pm 7.61)$ months. Two hundred seventy-nine (84.6\%) observations were censored at the end of the study. During the follow-up time, a total of 5609.54 person-months' time risk was observed with a minimum and maximum follow-up time of 1.2 and 27.5 months, respectively. Overall, 186 (56.4\%) women completed the program and transferred to lifelong ART follow-up, 27 (8.2\%) were transferred to other health institutions before completing the program (Fig. 1).

\section{Incidence and time to LTFU}

Of the total observations, 51 (15.4\%) women were LTFU by the end of the study with an overall incidence rate of LTFU of 9 per 1000 (95\% CI 6.8-11.9) person-months observations. The median time to LTFU from option B+ PMTCT program was 10.4 (95\% CI 8.03-13.01) months. The highest incidence of LTFU was observed at 12th, 13th and 25th months of enrollment (18.1/1000, $18.9 / 1000$ and $18.5 / 1000$ person-months) observations, respectively.

\section{Predictors of LTFU}

Variables considered for multivariable cox regression analysis were those with a $\mathrm{p}$-value $<0.25$ in bivariable analysis and 16 variables were selected in the first step of model buildings. After running backward stepwise variables selection process, the first group was selected as the best model from LLH ratio table, which include full (9) variables (educational level, residence, disclosure status, partner HIV status, status of women on initiation of ART, previous history of HIV, hemoglobin level, level of ART adherence and baseline regimen) in multivariable analysis.

Six of the predictors (educational level, residence, disclosure status, status of women on initiation of ART, previous history of HIV and level of ART adherence) were found to have statistically significant association with outcome variable and found to be independent predictors of the incidence of LTFU during multivariable cox proportional regression analysis at $95 \% \mathrm{CI}$. The risk of LTFU among women who had no education was 4.01 times higher than women who had secondary and above education level (AHR $=4.01,95 \%$ CI 1.84-8.76). Likewise, LTFU among women who were residing in the rural areas was 2.80 times higher than women who were residing in the urban areas $(\mathrm{AHR}=2.80,95 \%$ CI 1.54 5.08). The risk of LTFU among women who did not disclose their HIV status was 2.80 times higher than those women who disclosed their HIV status (AHR $=2.80,95 \%$ CI 1.50-5.22). Moreover, initiation of ART program and previous history of HIV showed a statistically significant association with LTFU. That is, the risk of LTFU among women who were enrolled to the program after delivery was 4.10 times higher than women who initiated the program during pregnancy $(\mathrm{AHR}=4.10,95 \%$ CI 2.03-8.28) and its risk among women who were newly diagnosed for HIV on entry to PMTCT or no previous history of HIV was 3.04 times higher than women who had the previous history of HIV (AHR $=3.04,95 \%$ CI 1.59-5.80). On last the follow-up, the risk of LTFU among women who had poor and fair level of adherence was 2.80 times higher

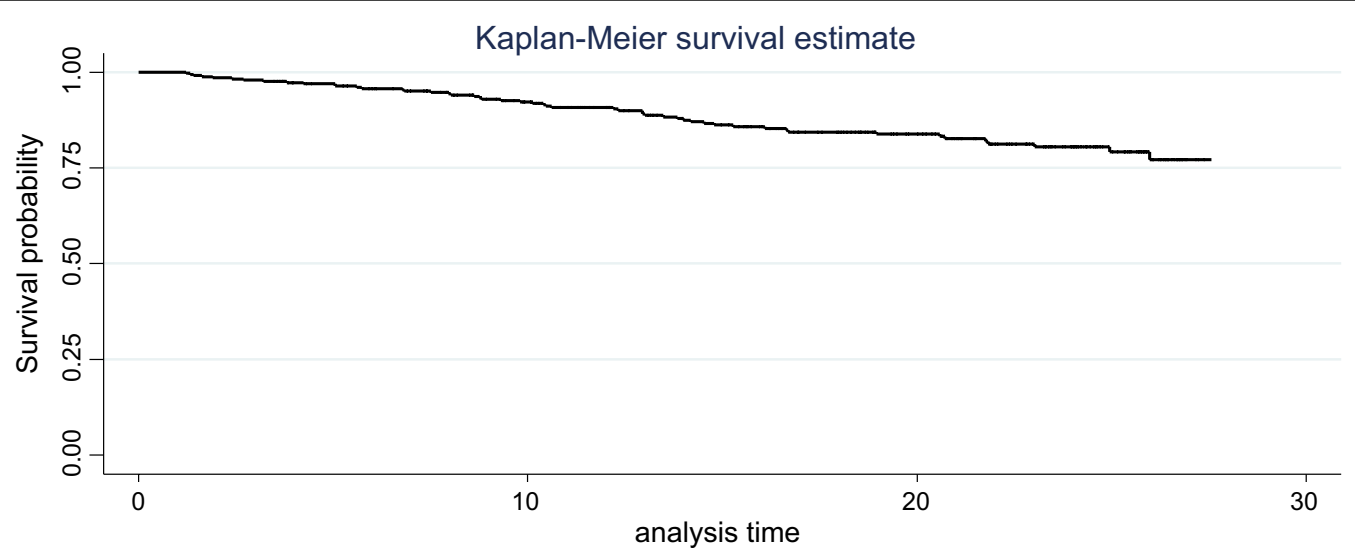

Fig. 1 The Kaplan-Meier survival function estimates of HIV positive women under option B+ PMTCT program at Nekemte Specialized Hospital, western Ethiopia, 2013-2019 
Table 2 Multivariable cox proportional analysis of incidence and predictors of LTFU among women under Option B+ PMTCT at Nekemte Specialized Hospital, western Ethiopia, 2013-2019

\begin{tabular}{|c|c|c|c|c|c|c|}
\hline \multirow[t]{3}{*}{ Variables } & \multirow[t]{3}{*}{ Category } & \multicolumn{2}{|c|}{ Survival status } & \multirow[t]{3}{*}{ CHR } & \multirow[t]{3}{*}{ AHR } & \multirow[t]{3}{*}{$p$-value } \\
\hline & & LTFU & Censored & & & \\
\hline & & No (\%) & No (\%) & & & \\
\hline \multirow[t]{3}{*}{ Educational level } & No education & $22(43.1)$ & $63(22.6)$ & $3.95(1.87-8.35)$ & $4.01(1.84-8.76)$ & $<0.001^{*}$ \\
\hline & Primary & $19(37.3)$ & $94(33.7)$ & $2.23(1.03-4.81)$ & $1.63(0.72-3.68)$ & 0.232 \\
\hline & Secondary and above & 10 (19.6) & $122(43.7)$ & 1 & 1 & \\
\hline \multirow[t]{2}{*}{ Residence } & Rural & $34(66.7)$ & $97(34.8)$ & $3.43(1.91-6.14)$ & $2.80(1.54-5.08)$ & $0.001^{*}$ \\
\hline & Urban & 17 (33.3) & $182(65.2)$ & 1 & 1 & \\
\hline \multirow[t]{2}{*}{ Disclosure status } & No & $29(56.9)$ & $56(20.1)$ & $4.15(2.38-7.24)$ & $2.80(1.50-5.22)$ & $0.001^{*}$ \\
\hline & Yes & $22(43.1)$ & $223(79.9)$ & 1 & 1 & \\
\hline \multirow[t]{3}{*}{ Partner HIV status } & Positive & $8(15.6)$ & $98(35.1)$ & 1 & 1 & \\
\hline & Negative & $24(47.1)$ & $113(40.5)$ & $2.25(1.01-5.01)$ & $2.27(0.97-4.87)$ & 0.059 \\
\hline & Unknown & $19(37.3)$ & $68(24.4)$ & $3.26(1.42-7.45)$ & $1.90(0.79-4.52)$ & 0.146 \\
\hline \multirow[t]{2}{*}{ Status of women on enrollment } & Pregnant & $39(76.5)$ & $249(89.2)$ & 1 & 1 & \\
\hline & Lactating & $12(23.5)$ & $30(10.8)$ & $3.62(1.84-7.09)$ & $4.10(2.03-8.28)$ & $<0.001^{*}$ \\
\hline \multirow[t]{2}{*}{ Previous history of HIV } & Yes & $20(39.2)$ & $201(75.7)$ & 1 & 1 & \\
\hline & No & $31(60.8)$ & $78(24.3)$ & $3.84(2.18-6.75)$ & $3.04(1.59-5.80)$ & $0.001^{*}$ \\
\hline \multirow[t]{2}{*}{ Hemoglobin } & $\leq 12.0$ & $19(37.3)$ & $143(51.3)$ & 1 & 1 & \\
\hline & $\geq 12.1$ & $32(62.7)$ & $136(48.7)$ & $1.67(0.95-2.96)$ & $1.77(0.97-3.25)$ & 0.061 \\
\hline \multirow[t]{2}{*}{ Recent adherence } & Good & $41(80.4)$ & $256(91.8)$ & 1 & 1 & \\
\hline & Fair/poor & 10 (19.6) & $23(8.2)$ & $2.40(1.20-4.80)$ & $2.80(1.29-6.07)$ & $0.009^{*}$ \\
\hline \multirow[t]{3}{*}{ Baseline regimen } & TDF/3TC/EFV & $42(82.4)$ & $198(80.0)$ & 1 & & \\
\hline & AZT/3TC/NVP & $6(11.8)$ & $45(16.1)$ & $0.60(0.25-1.43)$ & $1.11(0.43-2.85)$ & 0.815 \\
\hline & AZT/3TC/EFV & $3(5.8)$ & $36(12.9)$ & $0.40(0.12-1.29)$ & $0.44(0.12-1.52)$ & 0.198 \\
\hline
\end{tabular}

CHR crude hazard ratio, $A H R$ adjusted hazard

than those women who had a good level of ART adherence (AHR $=2.80,95 \%$ CI 1.29-6.07) (Table 2).

\section{Discussion}

This study was conducted to determine the incidence of LTFU and its predictors among women under the Option B+ PMTCT program. The overall incidence rate of LTFU from the Option B+ PMTCT program (9/1000) was lower than the finding from south Wollo study in northeast Ethiopia (14.8/1000 person-months) [16]. This discrepancy might be due to the time differences when the studies were conducted. The previous study was conducted when the program was initiated for the first time in the country, which might have increased the rate of LTFU due to lack of resources allocated including trained human power, to implement the program effectively. The finding is also lower than studies conducted in different countries such as Myanmar, Uganda, South Africa, Malawi and Kenya [12, 14, 17-19]. These variations might be due to the differences in definitions of LTFU in different settings as indicated above.

The study also identified different predictors of LTFU from the program. For instance, consistent with previous studies from low and middle income-countries (LMICs), the risk of LTFU among women with no education was higher than women who had attended secondary and above education $[12,20,21]$ where each additional year in school increases the likelihood of retaining to the program. Apparently, higher education levels contribute to better health literacy, self-care and greater access to information about the program. Moreover, consistent with findings Tanzania, Malawi and Uganda [15, 21, 22], the risk of LTFU among women residing in rural was higher as compared to women residing in urban, suggesting distance from home to hospitals, particularly in rural areas where women cannot easily get transport services due to physical barriers, forces women to walk long distances, may force them to miss appointments leading to poor adherence to ART.

HIV disclosure status significantly predicted LTFU in many low and middle-income countries $[12,13,21,23$, 24]. This might be related to the stigma associated with the disease, fear of negative consequences from their partners and perceiving to preserve family stability.

The risk of LTFU among women who were enrolled in the program during breastfeeding was higher than 
women who were enrolled in the program during pregnancy $[14,25]$. However, this finding is in contrast with studies conducted in Malawi in which the risk of LTFU from the Option $\mathrm{B}+$ program was higher in pregnant women than women who enrolled to program after delivery $[17,26]$. This discrepancy might be because of the difference on the date of ART initiation. In Malawi, most women start ART under Option B+ during pregnancy with the principle of "test and treat" approach. Hence, initiating ART on the same day with HIV diagnosis or under the "test and treat" approach increases the risk of LTFU from the program $[27,28]$.

The risk of LTFU among women who were newly diagnosed with HIV was consistently higher than women who were previously diagnosed with HIV [25, $29,30]$.

In summary, the highest incidence of LTFU was observed in the last month of follow up which implies poor linkage and referral systems between PMTCT and ART clinics. Thus, it is better to strengthen the linkage and referral system between PMTCT and ART clinics. Further prospective cohort and qualitative study should be conducted to identify the relationship between different identified factors and LTFU.

\section{Limitation of the study}

First, it is a retrospective review of records, which further limited due to unregistered outcomes and limited information regarding basic sociodemographic and clinical characteristics. Second, the true incidence of LTFU might be underestimated and/or overestimated due to the incomplete documentation of data and some of the participants classified as LTFU might have died or silently transferred to other health institutions.

\begin{abstract}
Abbreviations
AHR: adjusted hazard ratio; ART: antiretro viral therapy; $\mathrm{Cl}$ : confidence interval; CHR: crude hazard ratio; HIV: human immunodeficiency virus; LTFU: Iost to follow up; MTCT: mother to child transmission; PMTCT: prevention of mother to child transmission; SSA: sub-Saharan Africa; WHO: World Health Organization.
\end{abstract}

\section{Acknowledgements}

We would like to thank Wollega University for covering data collectors and supervisors per diem. Also, we would like to thank data collectors and supervisor.

\section{Authors' contributions}

TT has developed the proposal, participated in data collection supervision, data entry and data analysis. GMK, HC, AA, and DM has participated in proposal development and manuscript writing. All authors read and approved the final manuscript.

\section{Funding}

Wollega University has covered the costs of data collectors and supervisors per diem. The funded organization has no role in designing the study, data collection, or manuscript preparation.

\section{Availability of data and materials}

The datasets analyzed during the current study are available from the corresponding author upon reasonable request.

\section{Ethical approval and consent to participate}

Ethical clearance was obtained from Review Ethics Committee of College of Health Sciences, Debre Markos University. First formal letter was written from Debremarkos University to Nekemte specialized hospital. Letter of cooperation was obtained from Nekemte Specialized Hospital to Prevention of Mother to Child Transmission and Antiretro viral therapy clinics of Nekemte specialized hospital to conduct the study. Moreover, consent to participate was not applicable since it is record review and patient confidentiality was kept while the study was conducted through review of medical records.

\section{Consent for publication}

Not applicable.

\section{Competing interests}

The authors declare that they have no competing interests.

\section{Author details}

${ }^{1}$ Department of Public Health, Institute of Health Science, Wollega University, P.O.BOX: 395, Nekemte, Ethiopia. ${ }^{2}$ Department of Midwifery, College of Health Science, Debre Markos University, Debre Markos, Ethiopia. ${ }^{3}$ Maternal and Child Wellbeing Unit, African Population and Health Research Centre, Nairobi, Kenya. ${ }^{4}$ Department of Nursing, Institute of Health Science, Wollega University, Nekemte, Ethiopia.

Received: 3 October 2019 Accepted: 28 December 2019 Published online: 07 January 2020

References

1. MSF International AIDS Working Group. Prevention of mother-to-child transmission (PMTCT) of HIV Protocol, 2018. Geneva: Medecins Sans Frontiers; 2017.

2. United Nation Joint HIV/AIDS Prevention Program (UNAIDS). Closing gaps breaking barriers righting injustices. Geneva: Global Aids Update; 2018.

3. World Health Organization (WHO). Programmatic update use of antiretroviral drugs for treating pregnant women and preventing HIV infection in infants. Geneva: HIV/AIDs Program; 2012.

4. (WHO), WHO. Prevention of mother-to-child transmission (PMTCT) of HIV. Geneva: World Health Organization; 2016.

5. World Health Organization (WHO). Progress report on the global plan towards the elimination of new HIV infections among children and keeping their mothers alive. UNAIDS: Unicef; 2015. p. 2015.

6. O'Brien Lisa, et al. The incremental cost of switching from option B to Option B+ for the prevention of mother-to-child transmission of HIV. Bull World Health Organ. 2014;92:162-70.

7. Organization WH. Programmatic update: use of antiretroviral drugs for treating pregnant women and preventing HIV infection in infants: executive summary. Geneva: World Health Organization; 2012.

8. UNAIDS, unicef, and WHO. The incredible journey of the global plan towards the elimination of new HIV infections among children by 2015 and keeping their mothers alive. Geneva: World Health Organization; 2016.

9. (UNAIDS), U.N.J.H.A.P.P. Closing gaps breaking barriers righting injustices. Geneva: Global Aids Update; 2018.

10. unicef. Addressing the Global HIV Epidemic among pregnant women, mothers, children and adolescents UNICEF's Global HIV response 2018-2021. Geneva: UNICEF; 2018.

11. Bigirimana F, Owiredu MN, Nuwagira IB. Prevention of mother-to-child transmission technical update: implementing the 'treat all' approach among pregnant and breastfeeding women living with HIV in the who African region. Victoria: Bigirimana; 2016.

12. Kiwanuka G, Kiwanuka N, Muneza F. Retention of HIV infected pregnant and breastfeeding women on Option B+ in Gomba District, Uganda: a retrospective cohort study. BMC Infect Dis. 2018;18(1):p 533. 
13. Lumbantoruan C, et al. Understanding women's uptake and adherence in Option B+ for prevention of mother-to-child HIV transmission in Papua, Indonesia: a qualitative study. PLoS ONE. 2018;13(6):e0198329.

14. Kyaw K, Oo M, Kyaw N. Low mother-to-child HIV transmission rate but high loss-to-follow up among mothers and babies in Mandalay, Myanmar; a cohort study. PLoS ONE. 2017;12(9):e0184426.

15. Kweyamba M, et al. Loss to follow-up among HIV positive pregnant and lactating mothers on lifelong antiretroviral therapy for PMTCT in rural Uganda. Adv Public Health. 2018. https://doi.org/10.1155/2018/7540587.

16. Mitiku I, Arefayne M, Mesfin Y. Factors associated with loss to follow-up among women in option B+ PMTCT programme in northeast Ethiopia: a retrospective cohort study. J Int AIDS Soc. 2016;19(1):p 20662.

17. Tweya H, Gugsa S, Hosseinipour M. Understanding factors, outcomes and reasons for loss to follow-up among women in option B+ PMTCT programme in Lilongwe, Malawi. Trop Med Int Health. 2014;19(11):1360-6.

18. Karcher Heiko, Omondi Austin, Odera John. Risk factors for treatment denial and loss to follow-up in an antiretroviral treatment cohort in Kenya. Trop Med Int Health. 2007;12(5):687-94.

19. Dorina O. Timing of pregnancy, postpartum risk of virologic failure and loss to follow-up among HIV-positive women. Boston University; 2017. p. 1593-1602.

20. Mihratu TL. Adherence to option B+ and associated factors among pregnant women on pmtct services at public health facilities of East Shawa Zone, Oromia, Ethiopia. iMedPub. 2018. 2(1:2).

21. Hodgson I, Plummer ML, Konopka SN, Colvin CJ, Jonas E, et al. A systematic review of individual and contextual factors affecting art initiation, adherence, and retention for hiv-infected pregnant and postpartum women. PLoS ONE. 2014;9(11):e111421. https://doi.org/10.1371/journ al.pone.0111421.

22. Tweya $\mathrm{H}$, et al. Loss to follow-up before and after initiation of antiretroviral therapy in HIV facilities in Lilongwe Malawi. PLoS ONE. 2018. https:// doi.org/10.1371/journal.pone.0188488.
23. Gourlay A, et al. Barriers and facilitating factors to the uptake of antiretroviral drugs for prevention of mother-to-child transmission of HIV in sub-Saharan Africa: a systematic review. J Int AIDS Soc. 2013. https://doi. org/10.7448/IAS.16.1.18588.

24. Ebuy $\mathrm{H}$, Yebyo $\mathrm{H}$, Alemayehu M. Level of adherence and predictors of adherence to the Option B+ PMTCT programme in Tigray, northern Ethiopia. Int J Infect Dis. 2015;33:123-9.

25. Phillips T, et al. Disengagement of HIV-positive pregnant and postpartum women from antiretroviral therapy services: a cohort study. J Int AIDS Soc. 2014; 17:19242.

26. Landes M, et al. Characteristics and outcomes of women initiating ART during pregnancy versus breastfeeding in option B+ in Malawi. BMC Public Health. 2016. https://doi.org/10.1186/s12889-016-3380-7.

27. Chan K, et al. Same day HIV diagnosis and antiretroviral therapy initiation affects retention in Option B+ prevention of mother-to-child transmission services at antenatal care in Zomba District, Malawi. J Int AIDS Soc. 2016;19(1):20672

28. Tenthani $\mathrm{L}$, et al. Retention in care under universal antiretroviral therapy for HIV-infected pregnant and breastfeeding women ('Option B+') in Malawi. AIDS. 2014;28(4):589-98.

29. Cichowitz C, et al. Vulnerable at each step in the PMTCT care cascade high loss to follow up during pregnancy and the postpartum period in Tanzania. AIDS Behav. 2018. https://doi.org/10.1007/s10461-018-2298-8.

30. Alison SE, Jessica J, Blandina M. Patterns of HIV care clinic attendance and adherence to antiretroviral therapy among pregnant and breastfeeding women living with HIV in the context of Option B+ in Zimbabwe. J Acquir Immune Defic Syndr. 2017;75:198-206.

\section{Publisher's Note}

Springer Nature remains neutral with regard to jurisdictional claims in published maps and institutional affiliations.
Ready to submit your research? Choose BMC and benefit from:

- fast, convenient online submission

- thorough peer review by experienced researchers in your field

- rapid publication on acceptance

- support for research data, including large and complex data types

- gold Open Access which fosters wider collaboration and increased citations

- maximum visibility for your research: over $100 \mathrm{M}$ website views per year

At BMC, research is always in progress.

Learn more biomedcentral.com/submissions 